\title{
Zinner Snydrome: A Rare Cause of Recurrent Urinary Problems in Middle Aged Men: A Case Report and Review
}

\author{
Bajaj SK ${ }^{1 *}$ and Van Ahlen $\mathrm{H}^{2}$ \\ ${ }^{1}$ Department of Radiology, Klinikum Osnabrueck, Germany \\ ${ }^{2}$ Department of Urology, Klinikum Osnabrueck, Germany
}

*Corresponding author: Dr. Satish Bajaj, Consultant Radiologist, Department of Radiology, Klinikum Osnabrueck, Am Finkenhuegel 1 49076, Osnabrueck (Germany), Email: Satish. bajaj@klinikum-os.de

\section{Research Article}

Volume 4 Issue 3

Received Date: August 10, 2020

Published Date: September 15, 2020

DOI: $10.23880 /$ crij-16000178

\section{Abstract}

We report a rare case of mesonephric duct abnormality, presented in a young male suffering from recurrent urinary tract infections and perineal discomfort. MRI of the pelvis revealed a triad of renal agenesis, ipsilateral cystic transformation of the seminal vesical and ejaculatory duct obstruction. A well document MR case with its radiological and clinical relevance is discussed.

Keywords: Zinner Syndrome; Ejaculatory Duct Obstruction; Mesonephric Duct Anomalies; Magnet Resonance Imaging (MRI)

\section{Introduction}

Zinner Syndrome is a rare disorder of urogenital tract. It comprises a unique triad of unilateral renal agenesis, ipsilateral cystic transformation of the seminal vesicle and obstruction of the ejaculatory duct due to mullerian duct anomaly. The condition manifests itself in the third to fourth decade of life due to recurrent urinary tract infections or as a cause of infertility. We present a well-documented case of Zinner syndrome with its MRI features and the relevant clinical manifestations.

\section{Case Report}

A 21 years old male patient was referred to the MRI Unit of the radiology department from a practising Urologist with symptoms of pain on exertion around perineum and also radiating to the penis. He presented with a history of repeated urinary tract infections. Recent blood and urine tests were within normal limits. Ultrasound showed retrovesical cystic changes in the region of the seminal vesicle. Perirectal examination was normal. There was no history of abdominal or pelvic operations. Absence of the right sided kidney was known to the patient. MRI of the abdomen and pelvis was performed with $1.5 \mathrm{~T}$ Philips scanners with the standard abdomen protocol in our hospital.

T2w Turbospinecho sequence of the upper abdomen showed absence of right kidney (Figures 1a,1b) and no other abnormality. Cystic lesions observed on the right sided seminal vesicle with fluid/fluid level, suggestive of an intracystic haemorrhage (Figures $2 \mathrm{a}, 2 \mathrm{~b}$ ).The lesions showed continuity between the dilated proximal seminal vesicle and the ejaculatory duct and vas deference (Figures 3a,3b). On contrast sequences no neoplastic changes were observed (Figure 4). No free fluid in the pelvis was visible. This triad of findings was highly suggestive of Zinner Syndrome, a rare urogenital anomaly. 


\section{MR images}
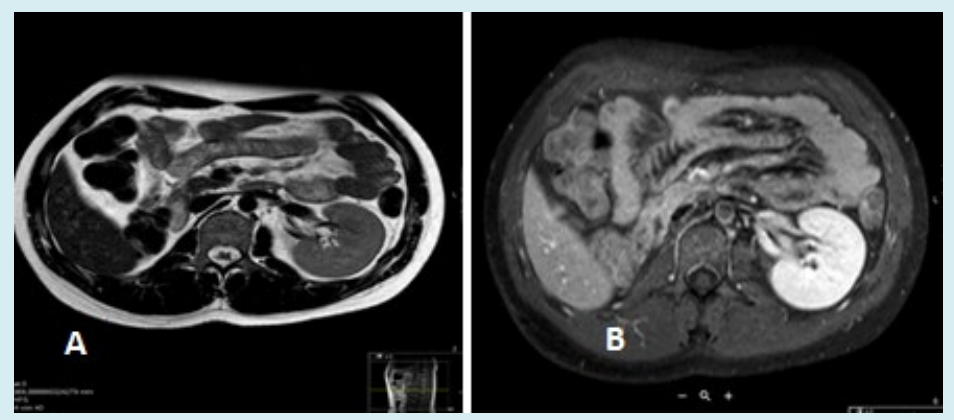

Figure1:(a)T2wTSEaxial(b)T1wTSEwithFatsuppressionwithcontrastmedia:showingabsenceofrightkidney,leftkidneynormal.
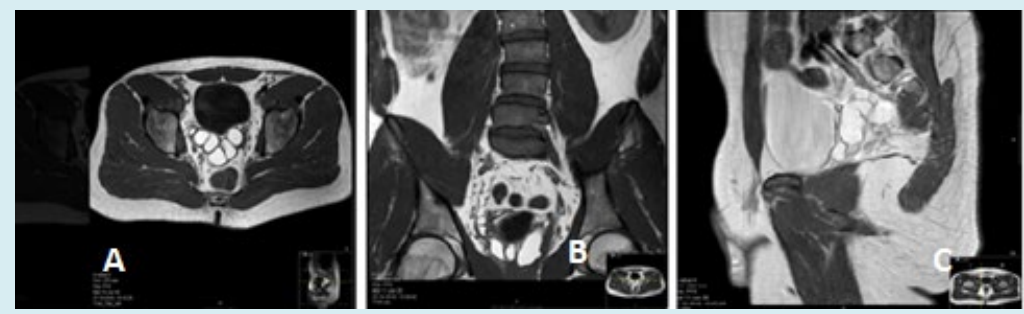

Figure 2: (a) T1 w TSE axial(a), coronal (b) and sagittal (c) showing cystic changes of the seminal vesicle and dilated ejaculatory duct on the right side (arrow).
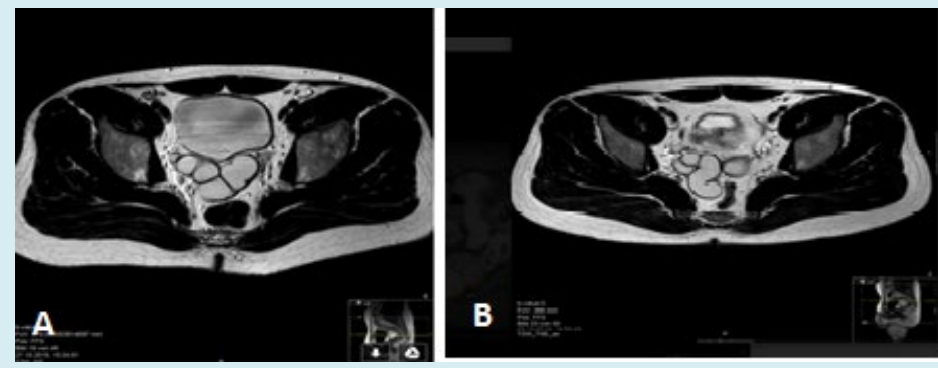

Figure 3 a, b: T2 w TSE axial showing cystic changes of the seminal vesicle (White arrow) without any evidence of infection or neoplasia.

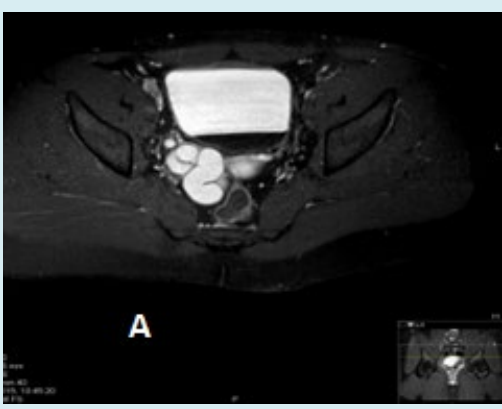

Figure 4: T1 w axial with Fat-suppression and Contrast showing cystic changes of the seminal vesicle without any enhancement.

\section{Discussion}

A spectrum of cystic lesions of lower genitourinary system has been reported in the literature with enormous diagnostic uncertainty, which may or may not cause any clinical signs and symptoms [1-3]. Understanding the anatomy and embryogenesis is of utmost importance in evaluation and proper diagnosis of these cystic disorders of the pelvis [4]. Mesonephric duct (Wolfian duct) arises as a paired organ during embryogenesis. In males, under the influence of testosterone and anti mullerian hormone $\mathrm{AMH}$; also known as Müllerian-inhibiting substance, "MIF" for "Müllerian-inhibiting factor", "MIH" for "Müllerian-inhibiting hormone", or "APH" for anti-paramesonephric hormone) 


\section{Clinical Radiology \& Imaging Journal}

$[5,6]$, it differentiates into the ipsilateral half of the trigone, bladder neck, urethra (distal up to external sphincter), seminal vesicle, vas deferens, efferent ducts, epididymis, and appendix epididymis [5-7].

Hence, any intrinsic or extrinsic factor altering the differentation of the mullerian duct will result into anomalies of above described organs of the urogenital tract. Maldevelopment of the distal end of mesonephric duct results in atresia of the ejaculatory duct with secondary cystic dilatation of the seminal vesical and also abnormal ureteral budding causing renal agenesis or dysgenesis. Abnormal cystic changes of the seminal vesicle and the ejaculatory duct leads to azoospermia/oligospermia resulting in infertility. Depending on the volume of the cystic, malformed seminal vesicle, pressure may be exerted on the surrounding region with secondary constant perineal pain and discomfort.

The triad of renal agenesis, ejaculatory duct obstruction and cystic changes of seminal vesicle was first described by Zinner in 1914 [8] and thereafter several cases have been reported [9]. MRI changes in Zinner syndrome have not been illustrated so far. Patients suffering from this syndrome are asymptomatic in early stages of life and slowly develop clinical symptoms during the peak of sexual and reproductive phase. They present with perineal or deep pelvic pain, dysuria, painful ejaculation, recurrent prostatitis, epididymitis and urinary tract infection. Large seminal vesicle cysts $(>12 \mathrm{~mm})$ may cause obstructive features of urinary bladder (urgency, prostatism) or colonic obstruction (defecation problems) $[10,11]$. Malignant transformation of the vesicle cyst is rare, but has been reported in the literature [12].

In our case, the patient was suffering from recurrent urinary tract infections with perineal discomfort. The ultrasound of the pelvis showed cysts of the seminal vesicle and he was referred to us for further evaluation with MRI examination. Ultrasound is a good screening tool in urological outpatients departments or private clinics. It may reveal kidney agenesis or dysgenesis. Seminal vesicle cysts are easily depicted by abdominal or transrectal sonography when the bladder is full. It can also delineate secondary changes within and in the walls of the cysts suggestive of infective or malignant processes $[13,14]$. Intravenous pyelography and CT scan is usually not performed for such an indication in young patient groups in Germany due to radiation hazards, even though such findings can be detected on routine CT examinations and may be labelled as incidental congenital findings and ignored without further evaluation [15].

MRI is the modality of choice for diagnosis in such cases $[16,17]$. It will accurately delineate cystic changes of the seminal vesicle with or without evidence of chronic infection and haemorrhage. Contrast studies will indicate features of malignant transformation like contrast enhancement of the wall or infiltrations of the surrounding soft-tissue with pathological enlarged lymph nodes in advanced cases. Coronal plane may show dilatation and obstruction of the ipsilateral vas deference. A spectrum of cystic disorders can be seen in the pelvic region including true cysts of the prostate gland, ejaculatory cysts, mullerian duct cysts and bladder diverticulum and duplication cysts. Hence on the basis of the location and morphology of the cyst along with other associated findings, a mullerian duct cyst can be differentiated from other cystic structures of the pelvis [18].

In conclusion, Zinner syndrome is a triad of mullerian duct anomaly with renal agenesis, ipsilateral seminal vesicle cysts and dilated ejaculatory duct due to obstruction in middle aged male patients, who present with recurrent urinary infections, infertility and pelvic discomfort. It should be considered in differential diagnosis, even though it is a very rare entity. MRI is the best diagnostic modality without any radiation hazards in this group of patients.

\section{References}

1. Kim B, Kawashima A, Ryu JA, Takahashi N, Hartman RP, et al. ( 2009) Imaging of the seminal vesicle and Vas deference. Radiographics 29(4): 1105-1121.

2. Ishikawa M, Okabe H, Oya T (2003) Midline prostatic cysts in healthy men: incidence and transabdominal sonography findings. AJR 181(6): 1669-1672.

3. Dirk P, Lock TM, Schrier BP, Zeijlemaker BY, Boon TA (1996) Transurethral marsupialization of medial prostatic cysts in patients with prostatitis like symptoms. J Urol 155(4): 1301-1304.

4. Sadler TW (2005) Urogenital system. In: Sadler TW Langman's essential medical embryology. 10 ${ }^{\text {th }}$ (Edn.) Philadelphia, Pa Lippincott Williams \& Wilkins.

5. Hannema SE, Huges IA (2007) Regulation of Wolfian duct development. Hormone Research in Paediatrics 67(3): 142-151.

6. Ball B, Conley A, Grundy S, Sabeur K, Liu I (2008) "Expression of anti-Mullerian hormone (AMH) in the equine testis". Theriogenology 69(5): 624-631.

7. Rey R, Grinspon R (2011) Normal male sexual differentiation and aetiology of disorders of sex development. Male Reproductive Endocrinology 25(2): 221-238.

8. Zinner A (1914) Ein fall von intravesikaler Samenblasenzste. Wien Med Wochensch 64: 605. 
9. Livingston L, Larson CR (2000) Seminal vesicle cyst with ipsilateral renal agenesis. AJR 175(1): 177-180.

10. Kneti J, Lissmer L, Smailowitz Z, Sober I (1988) Agenesis of kidney with malformation of the seminal vesicle, various clinical presentations. Int urol Nephrol 20(1): 29-33.

11. Heaney JA, Pfister RC, Meares EM (1987) Giant cyst of the seminal vesicle with renal agenesis. AJR 149(1): 139140 .

12. Okada Y, Tanaka H, Takeuchi H, Yoshida O (1992) Papillary adenocarcinoma in seminal vesicle cyst associated with ipsilateral renal agenesis: a case report. J Urol 148(5): 1543-1545.

13. Sheih CP, Hung CS, Wei CF, Lin CY (1990) Cystic dilatations within the pelvis in patient with ipsilateral renal agenesis or dysplasia. J Urol 144: 324-327.

14. Trigaux JP, Van Beers B, Delchambre F (1991) Male genital tract malformations associated with ipsilateral renal agenesis: sonographic findings. J Clin Ultrasound 19(1): 3-10.

15. Kenney PJ, Leeson MD (1983) Congenital anomalies of the seminal vesicles: spectrum of computed tomographic findings. Radiology 149(1): 247-251.

16. Arora SS, Breiman RS, Webb EM, Westphalen AC, Yeh BM, et al. (2007) CT and MRI of the congenital anomalies of the seminal vesicles. AJR 189: 130-135.

17. Murphy JO, Power RE, Aktar M, Torreggiani WC, McDermott TE, et al. (2003) Magnetic resonance imaging in the diagnosis of seminal vesicle cysts and associated anomalies. J Urol 170: 2386.

18. Shebel H, Farg HM, Kolokythas O, El-Diastty T (2013) Cysts of the lower male genitourinary tract: Embryologic and anatomic considerations and differential diagnosis. Radiographics 33(4): 1125-1143. 